\title{
Face Description with Monogenic Ternary Pattern: Application to Face Recognition
}

\author{
Yan He \\ College of Computer Science,Chongqing University of \\ Technology \\ No. 69 red road in Banan District of \\ Chongqing ,400054 China \\ cqyanhe@163.com
}

\author{
Wang Pu \\ College of Computer Science, Chongqing University of \\ Technology \\ No. 69 red road in Banan District of \\ Chongqing ,400054 China \\ 249195374 @qq.com
}

\begin{abstract}
This paper presents a new face description operator called monogenic ternary pattern (MTP) based on the theory of monogenic signal analysis and local ternary pattern ,then the histogram of MTP (HMTP) is subsequently and applied to the robust face recognition. HMTP consists of two parts:the first part is the monogenic magnitude encoded by local ternary pattern , and the other is monogenic orientation encoded by quadrant-bit codes. The HMTP is established by concatenating the histograms of MTP of all sub-regions. Extensive experimental results on YALE and FERET face databases show that the proposed MTP algorithm performs much better than state-of-the-art local feature methods .

Keywords-face recognition; monogenic signal analysis; local ternary pattern;
\end{abstract}

\section{INTRODUCTION}

Face recognition has an active development in the past few decades due to its potential applications in access control, intelligent surveillance, automated video surveillance, law enforcement and identity authentication, yet it is still an active research topic because of its many challenges in practice due to uncontrolled environments such as illumination, expression occlusions and variations in pose,etc[1]. There have been a lot of methods proposed for overcoming the difficulty of face recognition.These methods can be roughly divided into two groups:one is the global feature extraction method, such as the principal component analysis(PCA)[2]and the linear discriminant analysis(LDA)[3],etc, and the other is the local feature extraction method, such as scale invariant feature Transform[4], local binary pattern[5],etc.

LBP is a effective texture description operator[6] .Ahonen firstly used LBP for face recognition[7]. He calculates the binary relationship of each pixel in the image of its local neighborhood spot on gray scale; then the binary relationship weighted according to certain rules to form local binary patterns; finally, the histograms are used for face representation. This method showed a good performance on FERET face database, but the truth that directly calculating the LBP feature on the gray image will introduce a lot of noise .Based on LBP, Tan proposed local ternary pattern(LTP) which uses $0,1,-1$ to encode the face image and allows the user to customize a threshold that suppress the influence of noise effectively[8] .Follow LTP, LGTPHS [9] was proposed to use Gabor filtering to extract the histogram sequence of local Gabor ternary pattern which improves LTP'S robustness to noise and illumination changes. The Gabor phase was also used to improve the recognition rate [10]. Despite the great success of the above mentioned face recognition based on Gabor feature, the expense of Gabor feature methods is very expensive including both the storage space and the computational cost, because the Gabor filtering using 5-scale and 8-orientation to extract the facial feature which will leads to 80 convolutions for a single image .As a two dimensional generalization of one-dimensional analytic signal representation, monogenic signal representation decomposes an image into amplitude, phase, and orientation components, which represent the signal energetic, structural, and geometric information, respectively[11]. Different from Gabor transformation, monogenic signal representation does not use steerable filter to extract multiple-scale feature, and it has lower time and lower storage cost than Gabor filter.

This paper proposes a novel face description operator called monogenic ternary pattern (MTP) based on the theory of monogenic signal analysis and local ternary pattern, then using the histogram of HMTP for classification. HMTP consists of two parts:the first part is the monogenic magnitude encoded by local ternary pattern , and the other side is monogenic orientation encoded by quadrant-bit codes. MTP ingrates the advantages of monogenic signal analysis which can capture the energetic information and the geometric information,and the advantages of LTP which is robust to the changes of illumination and noise. Compared with the method based on the local feature method, The proposed method better performance. This is because that monogenic signal analysis is a compact representation of features with little information loss.

\section{MTP FOR FACE RECOGNITION}

\section{A. Monogenic signal analysis}

monogenic signal is a two-dimensional generalization of one-dimensional analytic signal representation, monogenic signal representation decomposes an image into amplitude, phase, and orientation components . It is built by the Riesz transform. The Riesz transform can be expressed as 


$$
\left(h_{x}, h_{y}\right)=\left(\frac{x}{2 \pi\|z\|^{3}}, \frac{y}{2 \pi\|z\|^{3}}\right)
$$

suppose the image is $z(x, y)$,then monogenic signal representation is defined as

$$
\begin{aligned}
& I_{M}(z)=\left(I, I_{x}, I_{y}\right)=\left(I, h_{x} * I, h_{y} * I\right) \\
& I=I(z) * F^{-1}(G(w))
\end{aligned}
$$

where “*” is the convolution operator, $G(w)$ is the Log-Gabor filter in Fourier domain, and it is defined as

$$
\begin{aligned}
& G(w)=\exp \left\{-\log \left(w / \omega_{0}\right)^{2} / 2\left[\log \left(\sigma / \omega_{0}\right)^{2}\right]\right\} \\
& \omega_{0}=\left(\lambda_{\min } \mu^{S-1}\right)^{-1}
\end{aligned}
$$

where $\omega_{0}$ is the center frequency and $\sigma$ is the scaling factor of the bandwidth. $\lambda_{\min }$ is the minimal wavelength, $\mu$ is the multiply factor of wavelength. $\mathrm{S}$ is the scale index. Then the face image can be expressed by monogenic amplitude, orientation, phase.They can be written as

$$
\begin{aligned}
& A=\sqrt{I^{2}+I_{x}^{2}+I_{y}^{2}} \\
& \theta=\arctan \left(\frac{I_{y}}{I_{x}}\right), \theta \in[0, \pi) \\
& P=\frac{-\operatorname{sign}\left(I_{x}\right)}{\arctan 2\left(\sqrt{\left(I_{x}^{2}+I_{y}^{2}\right)} / I\right)} ; P \in[0, \pi)
\end{aligned}
$$

\section{B. Face Represention based on MTP}

monogenic signal use amplitude $A$, orientation $\theta$, phase $P$ to describe the face image. The monogenic amplitude $A$ can describe local structure energy, high amplitude usually indicates higher energetic local features, and LTP is robust to noise and illumination changes. Therefore, the magnitude $A$ is encoded by LTP. The process of the magnitude $A$ is defined as follows:

$$
\begin{aligned}
& C_{A}\left(z_{c}\right)=\left[C_{p}^{A}, C_{p-1}^{A}, \ldots, C_{1}^{A}\right]_{\text {ternary }}=\left[\sum_{i=1}^{p} C_{i}^{A} 2^{i-1}\right] \\
& C_{i}^{A}\left(z_{c}\right)=\left\{\begin{array}{c}
1, A(i)-A\left(z_{c}\right) \geq t \\
0,-t \leq A(i)-A\left(z_{c}\right) \leq t \\
-1, A(i)-A\left(z_{c}\right)<-t
\end{array}\right.
\end{aligned}
$$

where $A\left(z_{c}\right)$ denotes the amplitude value of the center pixel $z_{c}$, and $A(i)$ denotes the amplitude value of the $i^{\text {th }}$ neighbor of the central pixel. $C_{A}\left(z_{C}\right)$ is the amplitude code, $t$ In order to reduce the computational complexity, we decomposed magnitude ternary code into two binary code as [7] does .we also use the uniform binary code, then the amplitude code map will be 6-bits at each scale.

Monogenic orientation describes the geometric information of the face image, which is an important feature in face recognition .In order to increase the robustness of Monogenic orientation coding, we adopt a quadrant bit to encode the monogenic orientation. It can be written as:

$$
C_{\theta}(z)=\left\{\begin{array}{l}
0, \text { if } h_{d}(z) \geq 0 \\
1, \text { if } h_{d}(z) \leq 0
\end{array} \quad, d \in\{x, y\}\right.
$$

where $h_{x}$ and $h_{y}$ are respectively the horizontal and vertical Riesz transform.Finally MTP is the combination of local orientation code and the amplitude code i.e. $M T P=\left(C_{\theta}^{x}, C_{\theta}^{y}, C_{A}(z)\right)$.After obtaining the MTP map, we need establish the histogram of MTP. Firstly, we need divide the MTP maps into multiple no-overlapping regions ,Then the histogram is established by all of the sub-regions. Finally, we concatenated all of the histogram built by the sub-regions into a vector to describe the face image.The HMTP can be written as

$$
H M T P=\left(H_{M T P}(h, s), h=1,2, \ldots, h ; s=1,2, \ldots, s\right)
$$

Where $h$ is the number of the sub-regions, $s$ is the scale of monogenic signal analysis. In the paper, The chi-distance[8] is used for classification and recognition.

\section{EXPERIMENTAL RESULTS}

In order to test the effectiveness of the proposed method, we perform an experiment on two benchmark face database. one is the YALE face database, and other one is FERET face database. The state-of-the-art local method based on LBP[], LTP[], and LGTPHS[] are used for comparison. some parameters need be setting, such as, multi-scale log-Gabor filtering and sub-region histogram. If no specific instruction, We set $\lambda_{\text {min }}=4, u=2, s=3, \sigma / \omega_{0}=0.65$. The face images are divided into $8 \times 8$ sub-regions in all the experiments.

\section{A. Results on the YALE database}

The YALE database includes 165 face images taken from 15 subjects, with each subject providing 11 face images. For some subjects, the image were taken at different times, with varying lighting,facial expressions(open/closed eyes, smiling/not smiling,occlusion/no-occlusion). In the experiment, we used one image of each subject with no variation in facial expressions as the training sample,and the remaining face images as training sample. The recognition rates and time cost of the experiments are listed in Table1.From the Table1,we can see that the time cost of the proposed MTP is slightly higher than LBP,LTP. However, its recognition rate is superior to LBP, LTP. LGTPHS have the some recognition rate as MTP, But its time cost is more higher than MTP . Our method has a good performance because that MTP ingrates the advantages of monogenic signal analysis which can capture a compact representation of features with little information loss, and 
the advantages of LTP which is robust to the changes of illumination and noise. LBP performs bad because that the binary code can be influenced by noise and illumination. LTP performs better than LBP because that it use ternary code and allow for the threshold. Despite the success of LGTPHS than LBP, LTP. Its time cost is very expensive.

Table 1. recognition rates(\%) and time cost(s) on the YALE database

\begin{tabular}{lcc}
\hline Methods & recognition rate & time cost \\
\hline LBP & 91.47 & 0.2463 \\
LTP & 97.33 & 0.3135 \\
LGTPHS & 99.54 & 12.345 \\
MTP & 99.54 & 1.256 \\
\hline
\end{tabular}

\section{B. results on the FERET database}

In the experiment, we use a subset of the FERET face database to test our method .This subset contains of 1400 images from 200 individuals.each providing 7 images. the image were taken by illumination and expression variations. We take the first three face images of each subject as training sample, and treated the remaining face images as testing sample. The recognition rates and time cost of the experiments are listed in Table2. From the Table2,we can see that The MTP we proposed have better performance than other methods.This is because MTP ingrates the advantages of monogenic signal analysis which can capture the energetic information and the geometric information,and the advantages of LTP which can extract effective local feature.LBP performs bad because the binary code can be influenced by noise and illumination. LTP performs better than LBP because that it use ternary code and allow for the threshold. Despite the success of LGTPHS than LBP, LTP. Its time cost is very expensive.

Table 2. recognition rates(\%) and time cost(s) on the FERET database

\begin{tabular}{lcc}
\hline Methods & recognition rate & time cost \\
\hline LBP & 75.38 & 0.2606 \\
LTP & 84.47 & 0.3414 \\
LGTPHS & 90.50 & 14.53 \\
MTP & 91.11 & 1.466 \\
\hline
\end{tabular}

\section{CONCLUSION}

The monigenic ternary pattern(MTP) proposed in this paper ingrates the advantages of monogenic signal analysis which can capture the energetic information and the geometric information,and the advantages of LTP which is robust to the changes of illumination and noise. After face extraction by MTP, the histogram of MTP (HMTP) is subsequently built and applied to the robust face recognition.HMTP consists of two parts:the first part is the monogenic magnitude encoded by local ternary pattern ,and the other side is monogenic orientation encoded by quadrant-bit codes. Experimental results on YALE and FERET database show that MTP performs better than LBP,LTP, LGTPHS. Despite the time cost of MTP is slightly higher than LBP,LTP.However its recognition rate is more higher. We do not make the most of the monogenic phase which will be exploited in our later work .

\section{ACKNOWLEDGEMENTS}

This work is supported by National Natural Science Foundation of China(NO. 61173184) and the Graduate Innovation Foundation of Chongqing University of Technology(NO. YCX2013219).

\section{REFERENCES}

[1] N. Poh, C.H. Chan, J. Kittler, S. Marcel, C.M. Cool, E.A. Rúa, J.L.A Castro, M. Villegas, R. Paredes, V. Struc, N. Pavesic, A.A. Salah, H. Fang, and N. Costen."An Evaluation of Video-to-Video Face Verification,” IEEE Trans. Information Forensics and Security, Vol. 5 , No. 4, 2010, pp. 781-801.

[2] M. Turk and A. Pentland. "Eigenfaces for recognition.” Journal of Cognitive Neuroscience,vol. 13,No. 1, 1991, pp. 71-86.

[3] S. W. Park and M. Savvides, “A multifactor extension of liner discriminant analysis for face recognition under varying pose and illumination,” EURASIP J. Adv. Signal Process, vol.2010, no.2,pp. 1116

[4] Krizaj J, Struc V, Pacesic N. “Adaptation of SIFT feature for face recognition”.ICAR 2010: Proceedings of the $7^{\text {th }}$ international Conference on Image Analysis and Recognition.Berlin:SpringerVerlag,2010, pp. :394-404

[5] T. Ojala, M. Pietikäinen, and D. Harwood. A comparative study of texture measures with classification based on feature distributions. PR, 1996, pp. 29:51-59,

[6] T. Ojala, M. Pietikäinen, and T. Mäenpää. Multile resolution gray-scale and rotation invariant texture classification with local binary patterns. IEEE TPAMI, 2002,vol. 24, no. 7, pp. 971 -987,

[7] Ahonen T, Hadid A, Pietikainen M. Face description with loca binary patterns : application to face recognition[J]. IEEE Trans Of Pattern Analysis and Machine Intelligence. 2006, vol. 28, no. 12. P:2037 -2041 .

[8] Tan Xiao-yang, Triggs B. "Enhanced local texture feature sets for face recognition under difficult lighting conditions. ” IEEE trans on Image Processing.vol. 19, no. 6, 2009, pp. 1635-1630.

[9] Zhang Jun, Zhang Qi-zhi,Zhou Ya-li,Xiang Yang,’Face Recognition based on Gabor ternary pattern.” Journal of beijing Information Science and Technology University, 2014, pp. 2099-2103.

[10] B. Zhang, S. Shan, X. Chen, and W. Gao. "Histogram of Gabor Phase Patterns (HGPP): A Novel Object Representation Approach for Face Recognition. IEEE TIP, vol. 16, no. 1,2006, pp. 57-68,

[11] YANG M, Lei Zhang and David Zhang."Monogenic Binary Pattern (MBP) : a novel feature extraction and representation model for face recognition.” Proceedings of the 20th IEEE International Conference on Pattern Recognition.Piscataway, NJ : IEEE Press, 2010, pp. 2680 2683 